\title{
Localized Orbitofrontal and Subcortical Metabolic Changes and Predictors of Response to Paroxetine Treatment in Obsessive- Compulsive Disorder
}

\author{
Sanjaya Saxena, M.D., Arthur L. Brody, M.D., Karron M. Maidment, R.N., \\ Jennifer J. Dunkin, Ph.D., Mark Colgan, M.E., Shervin Alborzian, \\ Michael E. Phelps, Ph.D., and Lewis R. Baxter, Jr., M.D.
}

Previous positron emission tomography (PET) studies of patients with obsessive-compulsive disorder (OCD) have found elevated glucose metabolic rates in the orbitofrontal cortex (OFC) and caudate nuclei that normalize with response to treatment. Furthermore, OCD symptom provocation differentially activates specific subregions of the OFC, which have distinct patterns of connectivity and serve different functions. Therefore, we sought to determine the role of specific subregions of the OFC and associated subcortical structures in mediating OCD symptoms, by determining how glucose metabolism in these structures changed with paroxetine treatment of OCD patients. We also sought to determine whether pretreatment OFC metabolism would predict response to paroxetine, as it has for other OCD treatments. Twenty subjects with OCD received $\left[{ }^{18} \mathrm{~F}\right]$-fluorodeoxyglucose (FDG)-PET scans before and after 8 to 12 weeks of treatment with paroxetine, $40 \mathrm{mg} /$ day. In patients who responded to paroxetine, glucose metabolism decreased significantly in right anterolateral OFC and right caudate nucleus. Lower pretreatment metabolism in both left and right OFC predicted greater improvement in OCD severity with treatment. These results add to evidence indicating that orbitofrontalsubcortical circuit function mediates the symptomatic expression of OCD. Specific subregions of the OFC may be differentially involved in the pathophysiology of OCD and/ or its response to pharmacotherapy.

[Neuropsychopharmacology 21:683-693, 1999] (C) 1999 American College of Neuropsychopharmacology. Published by Elsevier Science Inc.
From the University of California Los Angeles Department of Psychiatry and Biobehavioral Sciences (SS, ALB, KMM, JJD, LRB), University of California Los Angeles School of Medicine, Los Angeles, California; University of California Los Angeles Department of Molecular and Medical Pharmacology (MC, SA, MEP, LRB), University of California Los Angeles School of Medicine, Los Angeles, California; Department of Psychiatry and Behavioral Neurobiology (LRB), School of Medicine, University of Alabama at Birmingham, Birmingham, Alabama, USA.

Address correspondence to: Sanjaya Saxena, M.D., Assistant Professor, UCLA Department of Psychiatry and Biobehavioral Sciences, UCLA Neuropsychiatric Institute, 760 Westwood Plaza, Los Angeles, CA 90024-1759.

Received May 15, 1998; revised April 8, 1999; accepted July 8, 1999.
KEY WORDS: Obsessive-compulsive disorder (OCD);

Positron emission tomography (PET); Paroxetine; Cerebral glucose metabolism; Orbitofrontal cortex; Caudate, frontalsubcortical circuits

Previous positron emission tomography (PET) studies of obsessive-compulsive disorder (OCD) have found elevated glucose metabolic rates in the orbitofrontal cortex (OFC), anterior cingulate gyrus (AC), caudate nuclei, and thalamus (Baxter et al. 1987, 1988; Nordahl et al. 1989; Swerdlow 1995; see Saxena et al. 1998 for review) or cognitive-behavioral treatment (Baxter et al. 1992; Schwartz et al. 1996). Furthermore, interventions 
that provoke OCD symptoms have been found to increase blood flow to similar brain regions (McGuire et al. 1994; Rauch et al. 1994; Breiter et al. 1996). These and other findings have led to the theory that the symptomatic expression of OCD is mediated by hyperactivity along specific, frontal-subcortical circuits (Alexander et al. 1986) connecting the OFC, ventromedial caudate, globus pallidus, and the medial dorsal nucleus of the thalamus (Modell et al. 1989; Baxter et al. 1992; Baxter 1995; Insel 1992). Some studies have also suggested that hyperactivity in the $\mathrm{AC}$, which is also linked to basal ganglia structures and thalamus along a parallel circuit, may be involved in mediating some OCD symptoms (Rapaport and Wise 1988; Perani et al. 1995).

The OFC is a cytoarchitecturally heterogeneous structure that changes from agranular cortical tissue in its posteromedial region to more granular cortical tissue in its anterolateral region. These subregions have distinct patterns of connectivity and seem to serve different functions (see Zald and Kim 1996a; 1996b for detailed review). Previous PET studies of OCD treatment and symptom provocation suggest that different subregions of the OFC may be differentially involved in the pathophysiology of OCD. Benkelfat et al. (1990) found that the OFC subregion with the largest metabolic change with clomipramine treatment of OCD was right anterior OFC. McGuire et al. (1994) reported that OCD symptom intensity correlated with increased blood flow to right lateral OFC during symptom provocation. Rauch et al. (1994) found two centroids of OFC activation with symptomatic treatment-left anterior and right posterior OFC. Of these, only activation in left anterior OFC was positively correlated with the intensity of OCD symptoms; whereas, activation of right posterior OFC was negatively correlated with symptom intensity, suggesting that the two regions might play opposing roles in mediating and suppressing OCD symptoms, respectively. We sought to determine the role of specific subregions of the OFC and associated basal ganglia structures in mediating OCD symptoms, by determining how glucose metabolic rates in these structures changed with treatment of OCD patients. We sought to confirm and extend earlier findings using paroxetine hydrochloride, an OCD treatment that differs from those used in previous PET studies. Based on the previous findings noted above, we hypothesized that glucose metabolism in anterolateral OFC, caudate nucleus, and thalamus would preferentially decrease in responders to paroxetine.

Functional imaging data have also been examined to determine if pretreatment regional brain metabolism predicts treatment response. Swedo et al. (1989) found that responders to 2 months of clomipramine treatment had lower pretreatment absolute metabolic rates in right $\mathrm{OFC}$ and right $\mathrm{AC}$ than did nonresponders. Using data from our previous studies (Baxter et al. 1992;
Schwartz et al. 1996), our group found that pretreatment metabolic activity in left OFC differentially predicted response to cognitive-behavioral therapy (CBT) and fluoxetine in patients with OCD (Brody et al. 1998). Lower pretreatment left OFC-to-hemisphere ratio (LOFC/Hem) was significantly associated with better response to fluoxetine. In striking contrast, however, there was a significant association between higher pretreatment $\mathrm{LOFC} / \mathrm{Hem}$ and better response to CBT (Brody et al. 1998). Taken together, these results suggested that OCD patients with particular patterns of brain metabolism might respond preferentially to one type of treatment vs. the other (CBT vs. medication). Based on these findings, we sought to explore whether pretreatment metabolic activity in OFC would predict response to paroxetine treatment. We hypothesized that lower pretreatment $\mathrm{OFC} / \mathrm{Hem}$ would be correlated with greater eventual response to paroxetine, just as reported for clomipramine and fluoxetine treatment.

\section{SUBJECTS AND METHODS}

This study was carried out under guidelines established by the UCLA Human Subjects Committee. Written informed consent was obtained from all subjects after study procedures were fully explained. $\left[{ }^{18} \mathrm{~F}\right]-$ fluorodeoxyglucose (FDG)-PET images were obtained on 20 outpatients with OCD, 13 male and 7 female, before and after 8 to 12 weeks of treatment with paroxetine (40 mg/day), a serotonin reuptake inhibitor (SRI) medication found to be effective for OCD (Zohar and Judge 1996) and FDA approved for treatment of OCD. Subjects were recruited through advertisements placed in local newspapers, flyers, and referrals from a general outpatient intake center at the UCLA Neuropsychiatric Institute. All subjects were screened first by a research nurse coordinator (KMM) and then again by a treating psychiatrist (either ALB or SS) before study entry.

Diagnoses were made by clinical interview using DSM-IV criteria, and confirmed with the Schedule for Affective Disorders and Schizophrenia-Lifetime (SADS-L, Spitzer et al. 1978). Before treatment, each OCD patient had a Yale-Brown Obsessive-Compulsive Scale (Y-BOCS; Goodman et al. 1989) score greater than or equal to 16, and a 17-item Hamilton Depressive Rating Scale (HDRS; Hamilton 1960a) score under 10. All subjects were in good physical health. Two subjects had minor, concurrent motor or facial tics, but none met DSM-IV criteria for Gilles de la Tourette's syndrome. Subjects with other concurrent Axis I diagnoses, including major depression and substance abuse disorders or concurrent medical conditions that could affect brain function, were excluded. All subjects were free from psychoactive medication for at least 4 weeks before entering the 
study and free of fluoxetine for at least 5 weeks. Eight of the 20 subjects had never before received medication treatment for a psychiatric disorder. For the remaining 12 , the mean interval since their last medication treatment was 20 months (range 1-84 months). No psychoactive medications except paroxetine were allowed during the study period. All subjects received FDG-PET scans before and after treatment.

Subject symptom severity was rated with the Y-BOCS, HDRS, Hamilton Anxiety Scale (HAS, Hamilton 1960b), the Clinical Global Impression Scale (CGI), and the Global Assessment Scale (Endicott et al. 1976) at the time of each PET scan. All subjects were seen four to six times by the treating psychiatrist (either SS or ALB) during the study period for medication management, which consisted of paroxetine treatment titrated to a target dose of $40 \mathrm{mg} /$ day, as tolerated by the patient, and monitoring of side effects. Three subjects were unable to tolerate $40 \mathrm{mg}$ /day and were maintained on $30 \mathrm{mg}$ / day. "Responders" to treatment were defined a priori as those who had a greater than $25 \%$ drop in Y-BOCS score and a CGI rating of "much improved" or "very much improved" (as defined in our previous reportsBaxter et al. 1992; Schwartz et al. 1996). These criteria were chosen based on earlier use of these cutoffs for response in clinical studies (e.g., Montgomery et al. 1993; Wood et al. 1993; Koran et al. 1997). Patients who did not meet these response criteria were labeled "nonresponders" (Jenike and Rauch 1994).

\section{PET Methods}

FDG-PET methods were identical to those detailed in our previous report (Baxter et al. 1992) with the exception that scans began between 1 and 2 P.M. in this study. In brief, each subject received 5 to $10 \mathrm{mCi}$ of FDG while in supine position with eyes and ears open. After a 40min uptake period, emission scanning was performed for $40 \mathrm{~min}$, and each scan was reconstructed from two to three million counts. "Arterialized" venous blood was obtained by having the subject's hand in water-based hand warmer. Each subject's head was held in a special head holder during scanning to minimize head movement and allow accurate positioning in the tomograph. Scanning was performed on a Seimens-CTI PET tomograph (the ECAT III 831-15 transverse sections, spaced $6.75 \mathrm{~mm}$ apart, with 6-mm in-plane spatial resolution, acquired at an angle parallel to the cantho-meatal plane-for the first 14 subjects, and the EXACT HR1 961-47 transverse sections spaced 3.5-mm apart, with 4 $\mathrm{mm}$ in-plane spatial resolution-for the next six subjects). (Although spatial resolution differed between the two scanners, no difference was found between the first 14 subjects and the next six in pattern of cerebral metabolic changes with treatment-see Tables $2 a$ and $b$ ).
A region-of-interest (ROI) analysis was employed to determine regional changes in cerebral glucose metabolism with treatment. Image analysis involved co-registering each subject's pre- and post-treatment FDG-PET scans to correct for changes in head position between the scans, then identifying and outlining ROIs on each subject's pretreatment scan (see Figure 1) showing ROIs drawn below). This technique allowed standardization of ROIs across subjects and ensured that each volume outlined was the same for a given subject's pre- and post-treatment images, making the assessment of metabolic change in each ROI more accurate. PET to PET coregistration was performed using a 3-dimensional (3-D) image registration program (Lin et al. 1993). The co-registration program used the Powell algorithm for minimization with seven variable parameters (six for orientation adjustment and one for matching the scaling between the two image sets). The program then minimized the sum of the square of pixel value differences between image sets to align each subject's pre- and post-treatment PET images to one another (Lin et al. 1996).

Five bilateral ROIs were selected a priori, based on previous findings: (1) anterolateral orbitofrontal cortex (ALOFC); (2) posteromedial orbitofrontal cortex (PMOFC); (3) entire OFC; (4) head of the caudate nucleus; and (5) thalamus. We also examined an additional ROI, the AC, that was not postulated by us but by others. Both supratentorial hemispheres were also drawn so that ratios of ROI metabolism to ipsilateral hemisphere could be calculated to give normalized ratios (ROI/Hem). ROIs for OFC, caudate, thalamus, AC, and hemispheres were drawn in standardized fashion (Mazziota et al. 1983) using template sets previously obtained from normal anatomical and PET studies on the same tomograph, as described in our previous reports (Baxter et al. 1992; Schwartz et al. 1996). Previous ROI determinations in our laboratory have had very high inter-rater reliability (rI > 0.95; Small et al. 1992). For subregional analysis, the OFC was divided into two subregions of roughly equal size, one more anterolateral and one more posteromedial. The ALOFC included the anterior half of the middle orbital gyrus and lateral orbital gyrus, adjacent to the most anterior and inferior portion of Brodmann's area 10 (see Figure 1). The PMOFC included the medial orbital gyrus and Brodmann's area 47, but excluded the gyrus rectus, which formed its medial boundary.

ROIs were drawn by a technician (SA) blind to subject identity, diagnosis, or response, on pretreatment PET scans and then transferred onto co-registered posttreatment PET scans. Regional and hemispheric ROIs on each subject's pre- and post-treatment scans were compared to ensure that the scans were properly aligned, to eliminate misregistration artifacts. On each scan, ROIs were linked to give a summed value for the region. Ratios of each ROI normalized to ipsilateral 


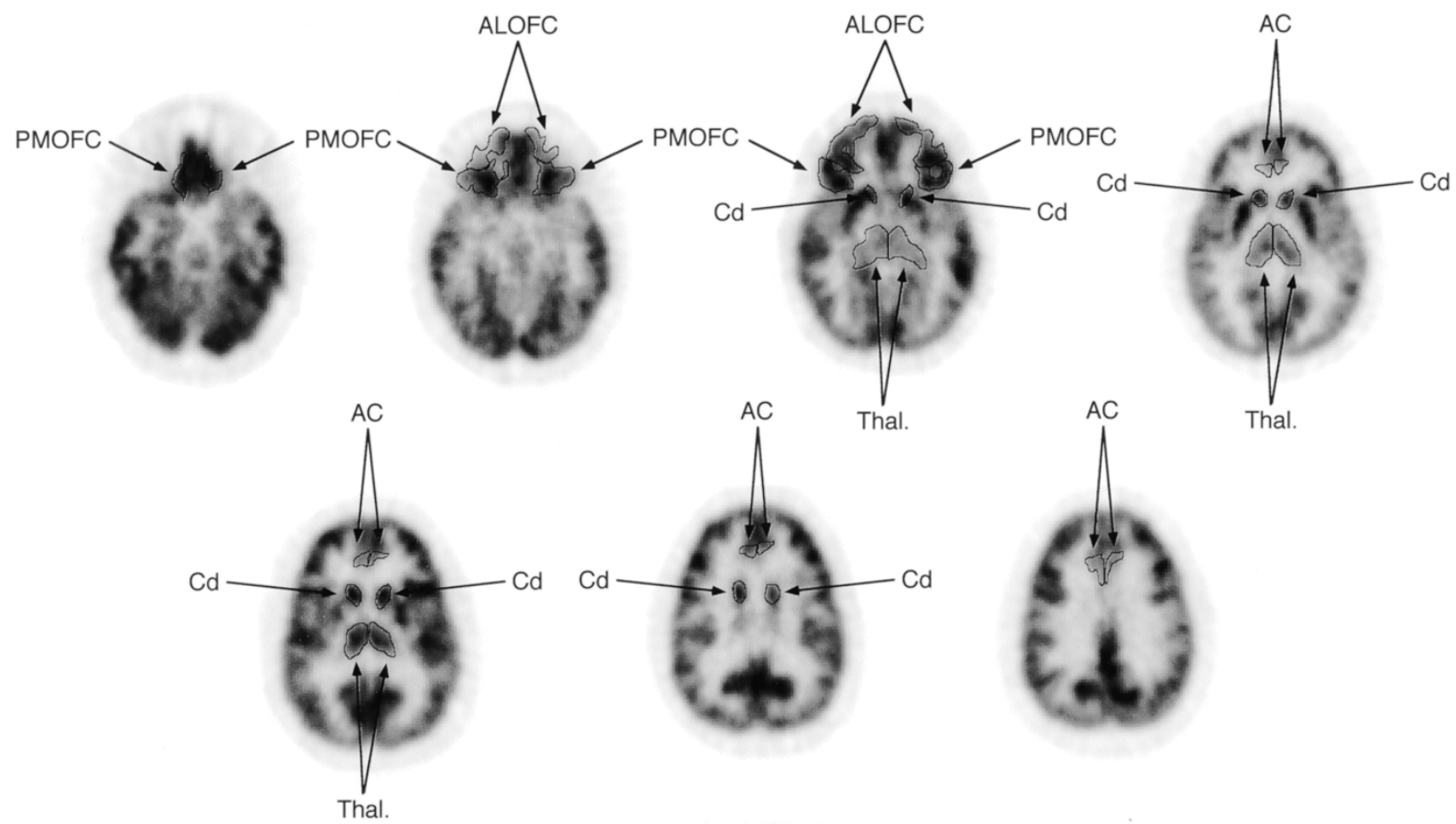

Figure 1. Regions of interest drawn on FDG-PET scans.

hemispheric glucose metabolism (ROI/Hem) were calculated as previously described (Baxter et al. 1992). We compared regional glucose metabolic changes between responders and nonresponders by performing a series of one-tailed, repeated measures analyses of variance (ANOVAs) with the normalized ROI/Hem values of each of the a priori, hypothesized regions (pre- and post-treatment) as the dependent variables, with time (pre- and post-treatment) as the within-subject factor, and treatment response status (responder vs. nonresponder) as the between-subject factor.

\section{RESULTS}

Eleven of the 20 subjects responded to paroxetine treatment (see Table 1 for rating scale scores). The proportion of responders scanned on the first scanner (eight of 14) was roughly the same as for the second scanner (three of six). Responders and nonresponders had similar pretreatment Y-BOCS scores $(26 \pm 6.2$ [mean $\pm \mathrm{SD}$ ] in responders versus $26 \pm 4.8$ in nonresponders), HDRS scores (9.3 \pm vs. $10 \pm 10 \pm 4.3)$, and HAS scores $(16 \pm 11$ vs. $18 \pm 9.6)$. Following paroxetine treatment, the responder group $(n=11)$ had a mean decrease in Y-BOCS score of $37 \pm 16 \%$; whereas, the nonresponder group $(n=9)$ had a mean decrease in Y-BOCS score of $5.8 \pm 17 \%$ (see Table 1 ).
ROI analysis (see Table 1) revealed a significant difference in the magnitude of change in right ALOFC/ Hem between responders (decreasing from $1.11 \pm .05$ pretreatment to $1.02 \pm .05$ post-treatment) and nonresponders (from $1.10 \pm .06$ pretreatment to $1.07 \pm .08$ post-treatment) [repeated measures analysis of variance (ANOVA), response $\times$ time $\mathrm{F}=4.99, \mathrm{df}=1 / 18, p=$ .04 , see Figure 2]. No differences were found between groups for changes in right or left PMOFC/Hem or left ALOFC/Hem, given that both groups showed similar decreases in metabolism in these subregions with treatment (see Table 1). Glucose metabolism in the entire OFC ROIs decreased bilaterally, but the difference between responders and nonresponders did not reach statistical significance.

Mean right caudate to hemisphere metabolic ration $(\mathrm{Cd} / \mathrm{Hem})$ decreased significantly in treatment responders (from $1.27 \pm .06$ to $1.22 \pm .05$ ) but not in nonresponders (from $1.23 \pm .04$ to $1.24 \pm .05$ ) (repeated measures ANOVA, response $\times$ time $\mathrm{F}=6.67, \mathrm{df}=1$ / $18, p=.01$, see Figure 2). The magnitude of change in left $\mathrm{Cd} / \mathrm{Hem}$ did not differ significantly between responders (decreasing from $1.25 \pm .06$ pretreatment to $1.23 \pm .08$ post-treatment) and nonresponders (no change in left $\mathrm{Cd} / \mathrm{Hem}$ ). Decreases in metabolism with treatment were seen in bilateral thalamus and $\mathrm{AC}$, but the magnitude of change did not differ significantly between responders and nonresponders in either structure. 
Table 1. Mean Normalized Metabolic Ratios (ROI/Hemisphere) and Rating Scale Scores

\begin{tabular}{|c|c|c|c|c|}
\hline \multirow[b]{2}{*}{ Mean Metabolic Ratio } & \multicolumn{2}{|c|}{$\begin{array}{l}\text { Responders } \\
(n=11)\end{array}$} & \multicolumn{2}{|c|}{$\begin{array}{l}\text { Nonresponders } \\
\quad(n=9)\end{array}$} \\
\hline & Pretreatment & Post-treatment & Pretreatment & Post-treatment \\
\hline Right ALOFC/Hem* & $1.11 \pm 0.05$ & $1.02 \pm 0.05$ & $1.10 \pm 0.06$ & $1.07 \pm 0.08$ \\
\hline Left ALOFC/Hem & $1.08 \pm 0.07$ & $1.02 \pm 0.06$ & $1.11 \pm 0.07$ & $1.06 \pm 0.08$ \\
\hline Right PMOFC/Hem & $1.13 \pm 0.05$ & $1.04 \pm 0.04$ & $1.14 \pm 0.06$ & $1.06 \pm 0.05$ \\
\hline Left PMOFC/Hem & $1.10 \pm 0.05$ & $1.03 \pm 0.06$ & $1.13 \pm 0.05$ & $1.06 \pm 0.07$ \\
\hline Right OFC/Hem & $1.11 \pm 0.04$ & $1.03 \pm 0.05$ & $1.13 \pm 0.06$ & $1.07 \pm 0.07$ \\
\hline Left OFC/Hem & $1.09 \pm 0.07$ & $1.02 \pm 0.07$ & $1.12 \pm 0.06$ & $1.06 \pm 0.08$ \\
\hline Right caudate/Hem** & $1.27 \pm 0.06$ & $1.22 \pm 0.05$ & $1.23 \pm 0.04$ & $1.24 \pm 0.05$ \\
\hline Left caudate/Hem & $1.25 \pm 0.06$ & $1.23 \pm 0.08$ & $1.25 \pm 0.05$ & $1.25 \pm 0.05$ \\
\hline Right thalamus/Hem & $1.12 \pm 0.06$ & $1.08 \pm 0.05$ & $1.16 \pm 0.08$ & $1.11 \pm 0.07$ \\
\hline Left thalamus/Hem & $1.13 \pm 0.06$ & $1.08 \pm 0.04$ & $1.15 \pm 0.05$ & $1.11 \pm 0.08$ \\
\hline Right AC/Hem & $1.14 \pm 0.04$ & $1.10 \pm 0.05$ & $1.13 \pm 0.09$ & $1.11 \pm 0.06$ \\
\hline Left AC/Hem & $1.15 \pm 0.04$ & $1.12 \pm 0.05$ & $1.10 \pm 0.06$ & $1.11 \pm 0.02$ \\
\hline \multicolumn{5}{|l|}{ Mean Rating Scale Score } \\
\hline Y-BOCS & $26 \pm 6.2$ & $16 \pm 5$ & $26 \pm 4.8$ & $24 \pm 7.5$ \\
\hline HDRS & $9.3 \pm 3.0$ & $7.1 \pm 1.9$ & $10 \pm 4.3$ & $9.6 \pm 6.5$ \\
\hline HAS & $16 \pm 11$ & $12 \pm 10$ & $18 \pm 9.6$ & $19 \pm 13$ \\
\hline
\end{tabular}

Difference between responders and nonresponders in change from pre- to post-treatment.

$* p=.04$.

$* * p=.01$.

Although subjects were scanned on two different PET tomographs (14 subjects on the Seimens ECAT III 831 tomograph and six subjects on the Seimens EXACT HR2 961 tomograph), their data were pooled for the primary data analysis. The proportion of responders scanned on the 831 (eight of 14) was similar to that for the 961 (three of six). Furthermore, analysis of the subjects scanned on the 831 vs. 961 tomograph showed no major differences in pattern of metabolic changes in responders vs. nonresponders (see Tables $2 \mathrm{a}$ and $\mathrm{b}$ ). The sample size of subjects scanned on the 961 tomograph was too small for separate statistical analyses.

Kendall's tau rank-order correlations were calculated between pretreatment metabolism in right and left OFC (the ROIs selected a priori for response prediction on the basis of previous findings) and response to paroxetine treatment, as measured by percentage change in Y-BOCs score. Because prediction of response was not this study's primary objective, the over-all study design did not lend itself to a definitive, prospective, placebocontrolled determination of cerebral metabolic predictors of response, only associations between pretreatment metabolic rate and treatment response. Therefore, these correlations were treated as secondary, exploratory analyses. Lower pretreatment left $\mathrm{OFC} / \mathrm{Hem}$ and lower pretreatment right $\mathrm{OFC} / \mathrm{Hem}$ were both significantly associated with better response to paroxetine $($ tau $=-.39, p=.01$ for left OFC $/ \mathrm{Hem}$-see Figure 3a and tau $=-.35, p=.02$ for right $\mathrm{OFC} / \mathrm{Hem}$ - see Figure $3 b$ ). [Exploratory analyses were also done for the four OFC subregions. These revealed an association between lower pretreatment left $\mathrm{PMOFC} / \mathrm{Hem}$ and better response to paroxetine (tau $=-.35, p=.03$ ) and trends toward correlation between pretreatment right PMOFC $/$ Hem and response (tau $=-.26, p=.08$ ), and between pretreatment left ALOFC/Hem and response (tau $=-.24, p=.09)$. However, these secondary correlations are not independent of those found for entire right and left OFC, and do not reach statistical significance after correlation for multiple comparisons].

\section{DISCUSSION}

To our knowledge, this is the first PET study of OCD subjects using ROIs drawn on co-registered pretreatment and post-treatment PET scans, ensuring that the neuroanatomical volumes outlined would be the same for both scans of each individual subject, making the assessment of metabolic change within each ROI more accurate. Utilization of a within-subjects design also reduced the possibility of discrepancy between pre- and post-treatment regions analyzed. Potential limitations of this study include the spatial resolution of the FDGPET method and variability of ROIs between subjects. Although previous ROI determinations in our laboratory have had high inter-rater ability $(\mathrm{rI}>0.95)$ (Small et al. 1992), there is still the possibility that slightly different regions were outlined for different subjects. For future studies, structural magnetic resonance imaging 


\section{Cerebral Metabolic Changes With Paroxetine Treatment of OCD}

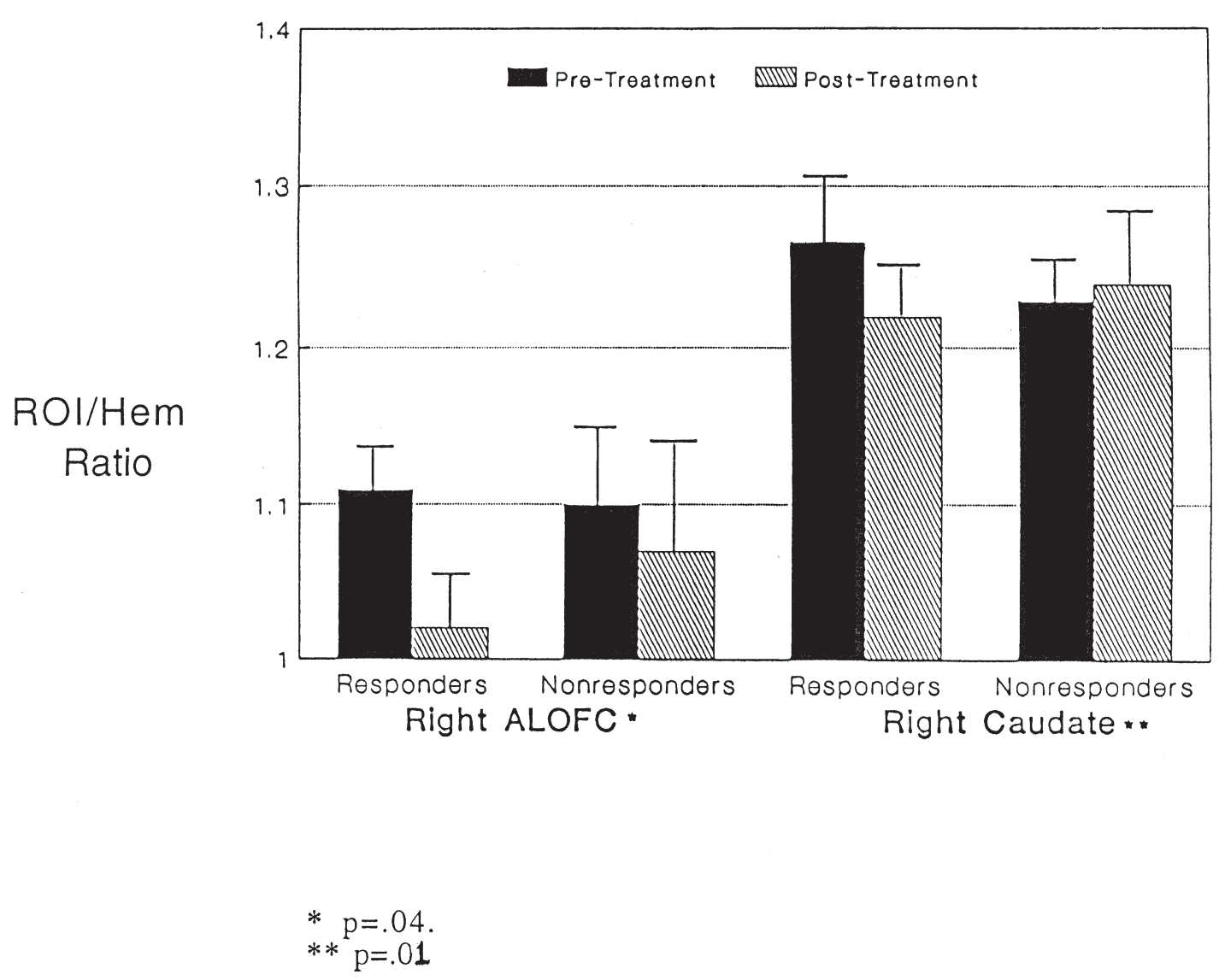

Figure 2. Mean pre- and post-treatment metabolic ratios $( \pm S D)$ in right ALOFC and right caudate, normalized to ipsilateral hemisphere (ROI/Hem), in responders vs. nonresponders to paroxetine. There was a significant difference in the magnitude of change in right ALOFC/Hem between responders (from $1.11 \pm .05$ pre-treatment to $1.02 \pm$ post-treatment) and nonresponders (from $1.10 \pm .06$ pre-treatment to $1.07 \pm .08$ post-treatment). Mean right Cd/Hem decreased significantly in treatment responders (from $1.27 \pm .06$ to $1.22 \pm .05$ ) but not in nonresponders (from $1.23 \pm .04$ to $1.24 \pm .05$ ). ${ }^{*} p=.04 ;{ }^{* *} p=.01$.

(MRI)-based localization of neuroanatomical regions will provide greater accuracy in standardizing ROIs across patients. Nevertheless, both intersubject variability in neuroanatomy and structural brain abnormalities in OCD subjects have been demonstrated in several structural neuroimaging studies (e.g., Robinson et al. 1995; Jenike and Rauch 1994; Rosenberg et al. 1997; Saxena et al. 1998 for review), making the drawing of ROIs on each subject's brain scan preferable for determining changes in specific brain regions than parametric mapping techniques that group subjects' brains together and align them to standardized coordinates based on the anatomy of small samples of control brains. Although such techniques as Statistical Parametric Mapping (SPM-Friston et al. 1991) are very useful for initially screening neuroimaging data, the ROI method may provide greater accuracy for determining metabolic changes in a priori neuroanatomical regions of interest in brains with abnormal morphology.
A further potential limitation of the study was the use of two different PET scanners (Seimens ECAT III 831 EXACT HR1 961). However, although the two scanners had different calibrations and spatial resolutions, there were no major differences in the metabolic changes seen in responders vs. nonresponders scanned on the 831 vs. the 961 (see Tables 2a and 2b). Furthermore, the proportion of responders scanned on the 831 (eight of 14) was similar to that for the 961 (three of six).

Consistent with the results of previous studies using other treatments, OCD patients in our study who responded to paroxetine had significantly greater glucose metabolic decreases in right OFC and right caudate nucleus than seen in nonresponders. Medication compliance was not verified with plasma drug levels in this study. This could potentially limit the specific interpretation of our results, because we cannot assume that our subjects' clinical improvement and cerebral metabolic changes resulted from paroxetine rather than nonmedi- 
Table 2a. Seimens ECAT III 831

\begin{tabular}{lccccc}
\hline & \multicolumn{2}{c}{$\begin{array}{c}\text { Responders } \\
(\boldsymbol{n}=\mathbf{8})\end{array}$} & & \multicolumn{2}{c}{$\begin{array}{c}\text { Nonresponders } \\
(\boldsymbol{n}=\mathbf{6})\end{array}$} \\
\cline { 2 - 3 } \cline { 5 - 6 } Mean Metabolic Ratio & Pretreatment & Post-treatment & & Pretreatment & Post-treatment \\
\hline Right ALOFC/Hem & $1.11 \pm 0.05$ & $1.02 \pm 0.05$ & & $1.09 \pm 0.06$ & $1.04 \pm 0.06$ \\
Left ALOFC/Hem & $1.07 \pm 0.08$ & $1.01 \pm 0.06$ & & $1.11 \pm 0.06$ & $1.04 \pm 0.05$ \\
Right PMOFC/Hem & $1.13 \pm 0.05$ & $1.05 \pm 0.04$ & & $1.13 \pm 0.05$ & $1.04 \pm 0.04$ \\
Left PMOFC/Hem & $1.10 \pm 0.05$ & $1.03 \pm 0.06$ & & $1.12 \pm 0.04$ & $1.02 \pm 0.04$ \\
Right OFC/Hem & $1.11 \pm 0.05$ & $1.03 \pm 0.06$ & & $1.11 \pm 0.05$ & $1.04 \pm 0.05$ \\
Left OFC/Hem & $1.09 \pm 0.07$ & $1.02 \pm 0.07$ & & $1.11 \pm 0.06$ & $1.03 \pm 0.04$ \\
Right caudate/Hem & $1.27 \pm 0.06$ & $1.23 \pm 0.06$ & & $1.23 \pm 0.04$ & $1.24 \pm 0.05$ \\
Left caudate/Hem & $1.24 \pm 0.06$ & $1.22 \pm 0.08$ & & $1.25 \pm 0.05$ & $1.25 \pm 0.05$ \\
Right thalamus/Hem & $1.12 \pm 0.06$ & $1.09 \pm 0.06$ & & $1.14 \pm 0.08$ & $1.10 \pm 0.06$ \\
Left thalamus/Hem & $1.11 \pm 0.04$ & $1.09 \pm 0.04$ & & $1.14 \pm 0.06$ & $1.10 \pm 0.09$ \\
Right AC/Hem & $1.13 \pm 0.04$ & $1.09 \pm 0.06$ & & $1.10 \pm 0.08$ & $1.10 \pm 0.06$ \\
Left AC/Hem & $1.13 \pm 0.03$ & $1.12 \pm 0.06$ & & $1.10 \pm 0.07$ & $1.10 \pm 0.02$ \\
Mean Rating Scale Score & & & & & \\
\hline Y-BOCS & $26 \pm 7.1$ & $16 \pm 6$ & & $26 \pm 3.8$ & \\
HDRS & $9.2 \pm 3.3$ & $6.8 \pm 1.8$ & & $10 \pm 5.0$ & $8.2 \pm 4.6$ \\
HAS & $16 \pm 11$ & $11 \pm 10$ & & $17 \pm 12$ & $20 \pm 14$ \\
\hline
\end{tabular}

Table 2b. Seimens EXACT HR1 961

\begin{tabular}{|c|c|c|c|c|}
\hline \multirow[b]{2}{*}{ Mean Metabolic Ratio } & \multicolumn{2}{|c|}{$\begin{array}{l}\text { Responders } \\
\quad(n=3)\end{array}$} & \multicolumn{2}{|c|}{$\begin{array}{l}\text { Nonresponders } \\
\quad(n=3)\end{array}$} \\
\hline & Pretreatment & Post-treatment & Pretreatment & Post-treatment \\
\hline Right ALOFC/Hem & $1.13 \pm 0.02$ & $1.08 \pm 0.01$ & $1.12 \pm 0.06$ & $1.13 \pm 0.07$ \\
\hline Left ALOFC/Hem & $1.10 \pm 0.04$ & $1.04 \pm 0.03$ & $1.12 \pm 0.09$ & $1.16 \pm 0.10$ \\
\hline Right PMOFC/Hem & $1.08 \pm 0.01$ & $1.03 \pm 0.04$ & $1.18 \pm 0.03$ & $1.09 \pm 0.05$ \\
\hline Left PMOFC/Hem & $1.10 \pm 0.02$ & $1.01 \pm 0.01$ & $1.18 \pm 0.05$ & $1.16 \pm 0.01$ \\
\hline Right OFC/Hem & $1.09 \pm 0.01$ & $1.05 \pm 0.02$ & $1.16 \pm 0.03$ & $1.13 \pm 0.05$ \\
\hline Left OFC/Hem & $1.09 \pm 0.02$ & $1.03 \pm 0.02$ & $1.14 \pm 0.04$ & $1.16 \pm 0.04$ \\
\hline Right caudate/Hem & $1.28 \pm 0.09$ & $1.22 \pm 0.04$ & $1.31 \pm 0.07$ & $1.29 \pm 0.08$ \\
\hline Left caudate/Hem & $1.25 \pm 0.06$ & $1.24 \pm 0.06$ & $1.27 \pm 0.08$ & $1.28 \pm 0.11$ \\
\hline Right thalamus/Hem & $1.12 \pm 0.07$ & $1.06 \pm 0.03$ & $1.18 \pm 0.08$ & $1.12 \pm 0.10$ \\
\hline Left thalamus/Hem & $1.14 \pm 0.04$ & $1.03 \pm 0.02$ & $1.16 \pm 0.04$ & $1.14 \pm 0.07$ \\
\hline Right $\mathrm{AC} / \mathrm{Hem}$ & $1.17 \pm 0.01$ & $1.11 \pm 0.01$ & $1.16 \pm 0.09$ & $1.12 \pm 0.08$ \\
\hline Left AC/Hem & $1.15 \pm 0.01$ & $1.11 \pm 0.02$ & $1.10 \pm 0.04$ & $1.12 \pm 0.02$ \\
\hline \multicolumn{5}{|l|}{ Mean Rating Scale Score } \\
\hline Y-BOCS & $24 \pm 1.7$ & $16 \pm 2.3$ & $26 \pm 6.8$ & $28 \pm 10$ \\
\hline HDRS & $9.7 \pm 2.1$ & $7.0 \pm 3.0$ & $9.0 \pm 3.2$ & $12 \pm 9.5$ \\
\hline HAS & $16 \pm 10$ & $11 \pm 9$ & $20 \pm 5.7$ & $23 \pm 15$ \\
\hline
\end{tabular}

cation factors or spontaneous remission. However, previous treatment studies have shown very low placebo rates in OCD (Wood et al. 1993; Jenike and Rauch 1994). Furthermore, our use of a within-subjects design allowed us to use each subject as his or her own control, assessing metabolic change associated with clinical improvement, regardless of what produced the improvement. Responders were compared to nonresponders to determine which changes were associated with symptomatic improvement. Our previous results demon- strated that similar metabolic changes occurred in responders to drug vs. behavior therapy (Baxter et al. 1992). The results of the present study confirm and extend the findings of prior studies that indicated that hyperactivity in orbitofrontal-basal ganglia-thalamocortical circuit mediated the symptomatic expression of OCD and that clinical improvement was associated with a decrease of this hyperactivity.

There is much experimental and clinical evidence that the OFC is involved in the mediation of emotional 


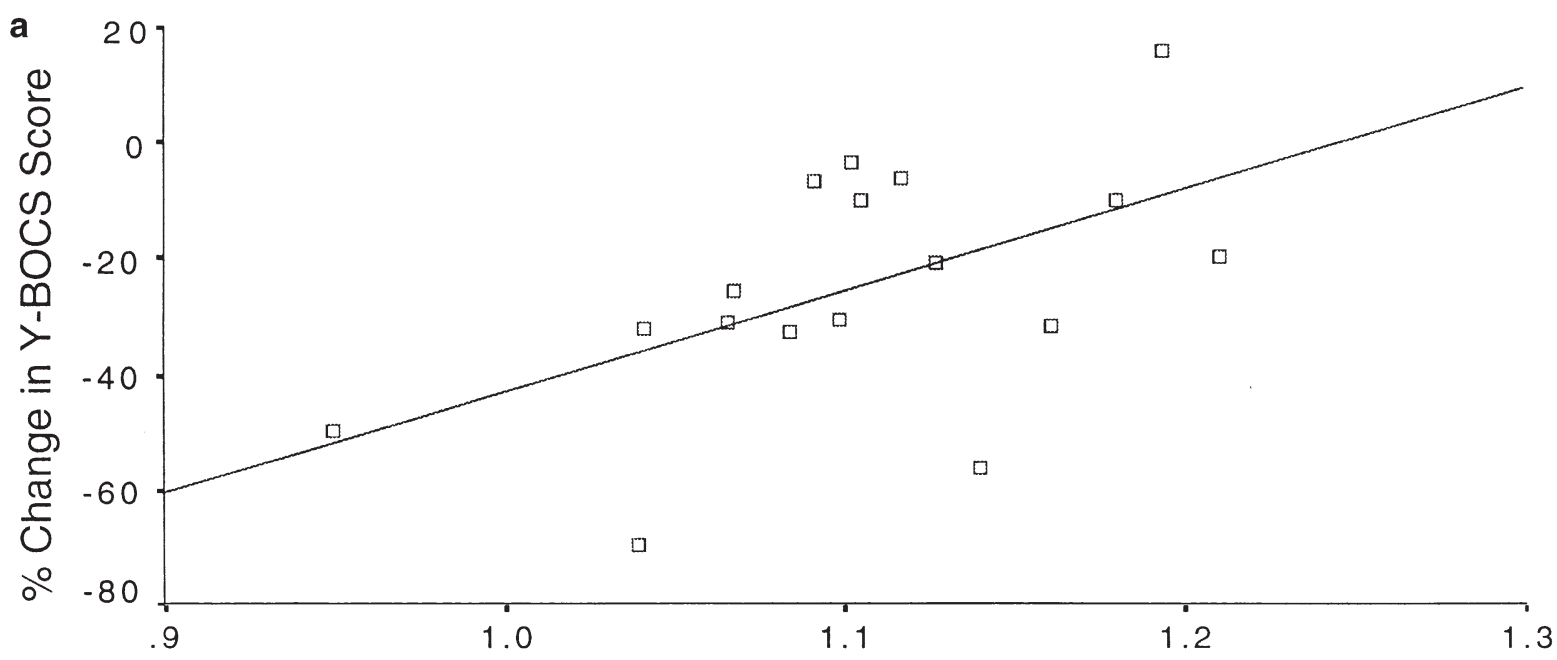

\section{Pre-Treatment Left OFC/Hem Ratio}

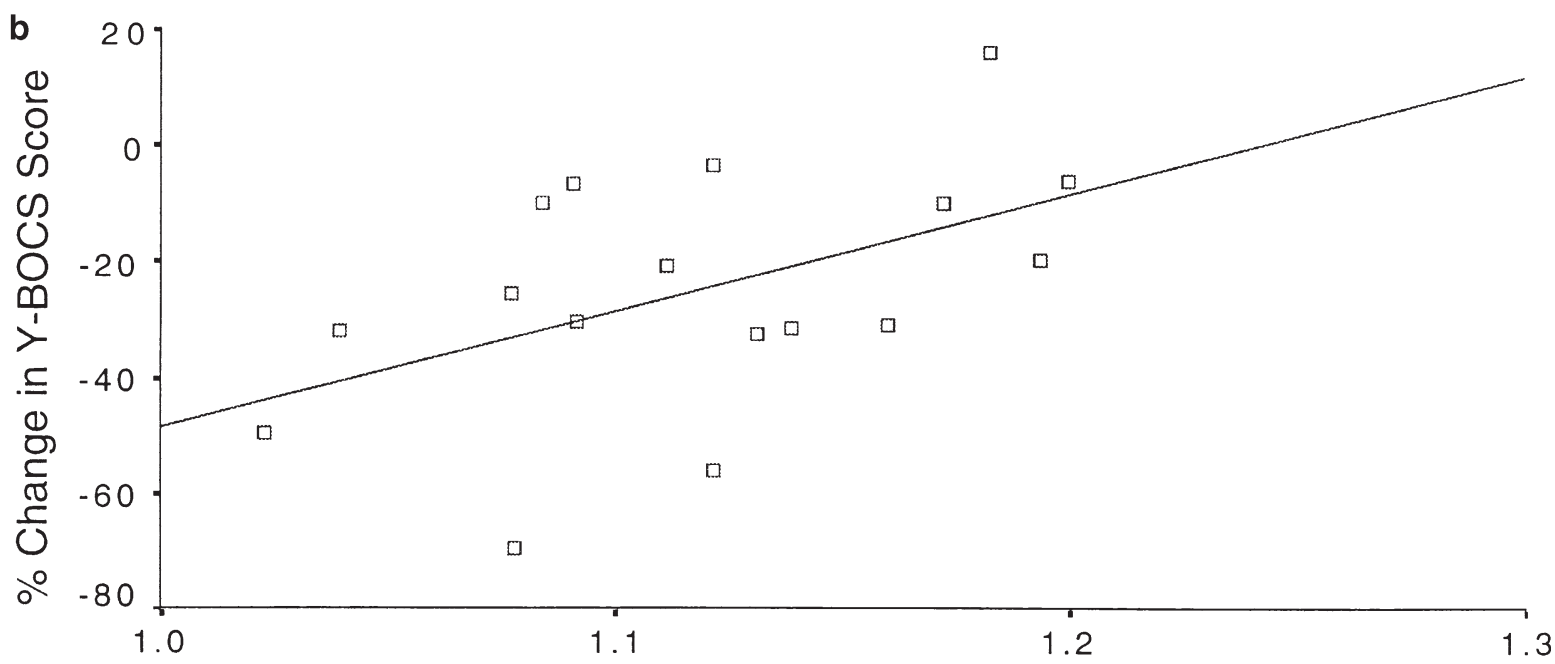

\section{Pre-Treatment Right OFC/Hem Ratio}

Figure 3a. Scatter plot of pretreatment glucose metabolic rate in left orbitofrontal cortex, normalized to ipsilateral hemisphere (left OFC/Hem), and change in Y-BOCS score after paroxetine treatment (Kendall's tau $=-.39, p=.01$ ).

Figure 3b. Scatter plot of pretreatment glucose metabolic rate in right orbitofrontal cortex, normalized to ipsilateral hemisphere (right OFC/Hem), and change in Y-BOCS score after paroxetine treatment (Kendall's tau $=-.35, p=.02$ ).

responses to biologically significant stimuli, as well as inhibition of behavioral responses (Zald and Kim 1996a, 1996b). In this study, responders to paroxetine had significantly greater localized metabolic decreases in the right anterolateral subregion of the OFC than did nonresponders, suggesting that this subregion may play a preferential role in the pathophysiology of OCD and/or its response to pharmacotherapy. This finding is consistent with previous findings of preferential decreases in right anterior OFC with response to clomipramine (Benkelfat et al. 1990) and increases in right lateral OFC with OCD symptom provocation (McGuire et al. 1994). Our results differ slightly from those of Rauch et al. (1994), who found activation of left, not right, ALOFC with symptom provocation. Aside from laterality, however, both our findings and those of Rauch et al. suggest that ALOFC may be more involved in mediating the active expression of OCD symptoms than PMOFC. Although PMOFC is nonspecifically activated by symptom provocation in a variety of anxiety disorders (Rauch et al. 1997), ALOFC might be more specifically involved in the pathophysiology of OCD and/or its re- 
sponse to pharmacotherapy. Alternatively, some have suggested that a reduction in OFC activity with improvement of OCD symptoms may reflect decreased "effort" required to resist intrusive thoughts and urges (Insel 1992), although there are no data directly correlating effortful resistance with OFC activity.

There is a large body of data delineating the neuroanatomical and functional differences between ALOFC and PMOFC (for review, see Zald and Kim 1996a, 1996b). PMOFC is a more paralimbic region with heavy connections to amygdala, insula, and entorhinal cortex; whereas, the more highly differentiated ALOFC has connections to associative neocortrical areas. Studies in lower primates have shown that POFC is required for direct stimulus-response learning and normal extinction of behavior (Butter 1969); whereas, ALOFC modulates behavior when there is more than one choice of responses and may be involved in nonspatial working memory (Goldman-Rakic 1987). Studies in normal humans suggest that ALOFC preferentially mediates alternation tasks (Gold et al. 1996). Patients with OCD have shown impaired performance on object alternation tasks (Abbruzzese et al. 1995; Cavedini et al. 1998), consistent with dysfunction of ALOFC and associated frontal-subcortical circuits.

\section{Response to Pharmacotherapy}

As in previous studies with other treatments, OCD patients in our study who responded to paroxetine had significantly greater glucose metabolic decreases in right OFC and right caudate nucleus than seen in nonresponders. These metabolic decreases may reflect decreased release of such excitatory neurotransmitters as glutamate in these regions (Moore et al. 1998). We and others have previously hypothesized that serotonin reuptake inhibitor (SRI) medications decrease activity in orbitofrontal-subcortical circuits, possibly by changing the relative balance of activity through the indirect vs. direct frontal-subcortical pathways (Swerdlow 1995; Baxter et al. 1996, 1999; Saxena et al. 1998) that exist within the associative and limbic cortical-basal gangliathalamocortical circuits (Smith et al. 1990; Shink et al. 1996; for review see Joel and Weiner 1997). Serotonin, by virtue of its pattern of projections to cerebral cortex and basal ganglia structures, is in a position to influence the balance between direct vs. indirect basal ganglia pathway activity, particularly for the associative an limbic frontal-subcortical circuits originating in the OFC and $\mathrm{AC}$. The serotonergic innervation of the striatum is heavily concentrated in the ventromedial caudata and nucleus accumbens, precisely those striatal subcompartments that receive input from the OFC and AC (Insel 1992; Parent and Hazrati 1995). Serotonergic pathways from the midbrain also project strongly to subthalamic nucleus and globus pallidus (Lavoie and
Parent 1990), key structures for the control of basal ganglia output in primates (Shink et al. 1996).

Serotonergic drugs may also ameliorate OCD symptoms by means of their effects in the OFC. Recent work has demonstrated differential effects of SRI drugs in orbitofrontal vs. dorsal prefrontal cortex, in a time course that corresponds to the effects of these medications on OCD symptoms vs. depressive symptoms. SRIs have been found both to enhance serotonin release and desensitize serotonin autoreceptors in the OFC after 8 weeks, but not 3 weeks; whereas, effects in the dorsal prefrontal cortex occur after 3 weeks (Mansari et al. 1995; Bergqvist et al. 1999). Given recent evidence by our group (Baxter et al. 1996) demonstrating in rodents that these two cortical regions have different effects on the direct and indirect basal ganglia pathways, the differential effects of serotonergic agents in the cerebral cortex alone could change the balance between direct vs. indirect pathway tone, resulting in the decrease in overall activity in OFC, AC, and caudate seen in functional imaging studies of OCD responders to treatment.

With paroxetine, a different treatment than used in previous neuroimaging studies of OCD, we replicated and extended previous findings (Swedo et al. 1989; Brody et al. 1998) of lower pretreatment OFC metabolism predicting response to clomipramine or fluoxetine. As hypothesized, patients with lower pretreatment left and right $\mathrm{OFC} / \mathrm{Hem}$ had a greater response to paroxetine. Of the OFC subregions, pretreatment left PMOFC metabolism had the strongest relationship with eventual response to paroxetine. These findings must be interpreted with caution, because they result from secondary, exploratory analyses. A more definitive determination of cerebral metabolic predictors of treatment response would require a randomized, prospective, placebo-controlled design with a larger sample size.

\section{ACKNOWLEDGMENTS}

This work was supported by the Charles A. Dana Foundation (Consortium on Neuroimaging Leadership Training), which provided funding for Dr. Saxena and Dr. Baxter; by NIMH grant R01 MH53565A to Dr. Baxter by the National Alliance for Research in Schizophrenia and Depression (NARSAD), which provided funding for Drs. Brody and Baxter, by Department of Energy grant \#DE FCE3-87ER 60615 to Drs. Phelps and Baxter, by donations from Mr. and Mrs. Brian Harvey, and by the Kathy Ireland Chair for Psychiatric Research at University of Alabama at Birmingham.

\section{REFERENCES}

Abbruzzese M, Bellodi L, Ferri S, Scarone S (1995): Frontal lobe dysfunction in schizophrenia and OCD: A neuropsychological study. Brain Cognit 27: 202-212 
Alexander GE, DeLong MA, Strick PL (1986): Parallel organization of functionally segregated circuits linking basal ganglia and cortex. Ann Rev Neurosci 9:357-381

Baxter LR (1995): Neuroimaging studies of human anxiety disorders: Cutting paths of knowledge through the field of neurotic phenomena. In Bloom F, Kupfer D (eds), Neuropsychopharmacology: The Fourth Generation of Progress. New York, Raven Press, pp 1287-1299

Baxter LR, Phelps EM, Mazziotta JC, Guze BH, Schwartz JM, Selin CE (1987): Local cerebral glucose metabolic rates in obsessive-compulsive disorder-A comparison with rates in unipolar depression and in normal controls. Arch Gen Psychiat 44: 211-218

Baxter LR, Schwartz JM, Mazziotta JC, Phelps ME, Pahl JJ, Guze BH, Fairbanks L (1988): Cerebral glucose metabolic rates in nondepressed obsessive-compulsives. Am J Psychiat 145: 1560-1563

Baxter LR, Schwartz JM, Bergman KS, Szuba MP, Guze BH, Mazziotta JC, Alazraki A, Selin CE, Ferng HK, Munford $P$ (1992): Caudate glucose metabolic rate changes with both drug and behavior therapy for obsessive-compulsive disorder. Arch Gen Psychiat 49:681-689

Baxter LR, Saxena S, Brody AL, Ackermann RF, Colgan M, Schwartz JM, Allen-Martinez Z, Fuster JM, Phelps ME (1996): Brain mediation of obsessive-compulsive disorder symptoms: Evidence from functional brain imaging studies in the human and nonhuman primate. Semin Clin Neuropsychiat 1:32-47

Baxter LR, Ackermann RF, Swerdlow NR, et al. (1999): Specific brain system mediation of OCD responsive to either medication or behavior therapy. In Goodman W, Rudorfer M, Maser J (eds), NIMH-OC Foundation Symposium Monograph. Bethesda, ME, National Institute of Mental Health

Benkelfat C, Nordahl TE, Semple WE, King AC, Murphy DL, Cohen RM (1990): Local cerebral glucose metabolic rates in obsessive-compulsive disorder. Patients treated with clomipramine. Arch Gen Psychiat 47:840-848

Bergqvist PB, Bouchard C, Blier P (1999): Effect of long-term administration of antidepressant treatments on serotonin release in brain regions involved in obsessive-compulsive disorder. Biol Psychiat 45:164-74

Breiter HC, Rauch SL, Kwong KK, Baker JR, Weiskoff RM, Kennedy DN, Kendrick AD, Davis TL, Jiang A, Cohen MS, Stern CE, Belliveau JW, Baer L, O'Sullivan RM, Savage CR, Jenike MA, Rosen BR (1996): Functional magnetic resonance imaging of symptom provocation in obsessivecompulsive disorder. Arch Gen Psychiat 53:595-606

Brody AL, Saxena S, Schwartz JM, Stoessel PW, Maidment KM, Phelps ME, Baxter LR (1998): FDG-PET predictors of response to behavioral therapy versus pharmacotherapy in obsessive-compulsive disorder. Psychiat Res: Neuroimag 84:1-6

Butter CM (1969): Perseveration in extinction and in discrimination reversal tasks following selective frontal ablations in Macaca mulatta. Physiol Behav 68: 558-561

Cavedini P, Ferri S, Scarone S, Bellodi L (1998): Frontal lobe dysfunction in obsessive-compulsive disorder and major depression: A clinical-neuropsychological study. Psychiat Res 78:21-8

Endicott J, Spitzer RL, Fleiss JL, Cohen J (1976): The global assessment scale. A procedure for measuring overall severity of psychiatric disturbance. Arch Gen Psychiat 41:586-601

Friston K, Frith C, Liddle P, Frackowiak R (1991): Comparing functional (PET) images: The assessment of significant change. J Cereb Blood Flow Metab 11:690-699

Gold JM, Berman KF, Randolph C, Goldberg TE, Weinberger DR (1996): PET validation of a novel prefrontal task: Delayed response alternation. Neuropsychology 10:3-10

Goldman-Rakic PS (1987): Circuitry of primate prefrontal cortex and regulation of behavior by representational memory. In Plum F, Mountcastle V (eds), Handbook of Physiology: The Nervous System. Bethesda, MD, American Physiological Society, pp 373-417

Goodman WK, Price LH, Rasmussen SA, Mazure C, Delgado P, Heninger GR, Charney DS (1989): The Yale-Brown obsessive-compulsive scale I. Development, use, and reliability. Arch Gen Psychiat 46:1006-1011

Hamilton M (1960a): Diagnosis and rating scale far depression. Brit J Psychiat 3:76-79

Hamilton M (1960b): A rating scale for anxiety. J Neurol Neurosurg Psychiat 23:56-62

Insel TR (1988): Obsessive-compulsive disorder: A neuroethological perspective. Psychopharm Bull 24:365-369

Insel TR (1992): Toward a neuroanatomy of obsessive-compulsive disorder. Arch Gen Psychiatry 49:739-744.

Jenike MA, Rauch SL (1994): Managing the patient with treatment-resistant obsessive-compulsive disorder. Current strategies. J Clin Psychiat 55:11-17

Joel D, Weiner I (1997): The connections of the primate subthalamic nucleus: Indirect pathways and the open-interconnected scheme of basal ganglia-thalamocortical circuitry. Brain Res Rev 23:63-78

Koran LM, Sallee FR, Pallanti S (1997): Rapid benefit of pulse-loading of clomipramine in obsessive-compulsive disorder. Am J Psychiat 154:396-401

Lavoie B, Parent A (1990): lmmunohistochemical study of the serotoninergic innervation of the basal ganglia in the squirrel monkey. J Comp Neurol 299: 1-16

Lin KP et al. (1993): A general technique for interstudy registration of multifunction and multimodality images. J Cereb Blood Flow Metab 9:96-103

Lin KP, Huang SC, Yu DC, Melega W, Barrio JR, Phelps ME (1996): Automated image registration for FDOPA PET studies. Phys Med Biol 41:2775-2788

Mansari ME, Bouchard C, Blier P (1995): Alteration of serotonin release in the guinea pig orbito-frontal cortex by selective serotonin reuptake inhibitors. Neuropsychopharmacology 13:117-127

Mazziota JC, Phelps ME, Plummer D (1983): Optimization and standardization of anatomical data in neurobehavioral investigations using positron emission tomography. J Cereb BF Metab 3:S266-S267

McGuire PK, Bench CJ, Frith CD, Marks IM, Frackowiak RS, Dolan RJ (1994): Functional anatomy of obsessive-compulsive phenomena. Brit J Psychiat 164:459-468

Modell JG, Mounts JM, Curtis GC, Greden JF (1989): Neurophysiological dysfunction in basal ganglia/limbic stri- 
atal and thalamocortical circuits as a pathogenetic mechanism of obsessive-compulsive disorder. J Neuropsychiat Clin Neurosci 1:27-36

Montgomery SA, McIntyre A, Osterheider M, Sarteschi P, Zitterl W, Zohar J, Birkett M, Wood AJ (1993): A doubleblind, placebo-controlled study of fluoxetine in patients with DSM-II-R obsessive-compulsive disorder. Euro Neuropsychopharmacol 3:143-152

Moore GJ, MacMaster FP, Stewart C, Rosenberg DR (1998): Case study: Glutamatergic changes with paroxetine therapy for pediatric obsessive-compulsive disorder. J Am Acad Child Adolesc Psychiat 37:663-667

Nordahl TE, Benkelfat C, Semple WE, Gross M, King AC, Cohen RM (1989): Cerebral glucose metabolic rates in obsessive-compulsive disorder. Neuropsychopharmacology 2: 23-28

Parent A, Hazrati L -N (1995): Functional anatomy of the basal ganglia. I. The cortico-basal ganglia-thalamocortical loop. Brain Res Rev 20:91-127

Perani D, Colombo C, Bressi S, Bonfanti A, Grassi F, Scanne S, Bellodi L, Smeraldi E, Fazio F (1995): [18F]FDG-PET study in obsessive-compulsive disorder: A clinical/ metabolic correlation study after treatment. Brit J Psychiat 166:244-250

Rapaport JL, Wise SP (1988): Obsessive-compulsive disorder: Is it a basal ganglia dysfunction? Psychopharm Bull $1: 27-36$

Rauch SL, Jenike MA, Alpert NM, Baer L, Breiter HC, Savage CF, Fischman AJ (1994): Regional cerebral blood flow measured during symptom provocation in obsessive-compulsive disorder using oxygen 15-labeled carbon dioxide and positron emission tomography. Arch Gen Psychiat 51:62-70

Rauch SL, Savage CR, Alpert NM, et al. (1997): The functional neuroanatomy of anxiety: A study of three disorders using positron emission tomography and symptom provocation. Biol Psychiat 42:446-52

Robinson D, Wu H. Munne RA, Ashtari M, Alvir JMJ, Lerner G, Koreen A, Cole K, Bogerts B (1995): Reduced caudate volume in obsessive-compulsive disorder. Arch Gen Psychiat 52:393-398

Rosenberg DR, Keshavan MS, O'Hearn KM, Dick EL, Bagwell WW, Seymour AB, Montrose DM, Pierri JN, Birmaker (1997): Frontostriatal measurement in treatmentnaive children with obsessive-compulsive disorder. Arch Gen Psychiat 54:82-830

Sawle GV, Hymas NF, Lees AJ, Frackowiak RSJ (1991):
Obsessional slowness: Functional studies with positron emission tomography. Brain 114:2191-2202

Saxena S, Brody AL, Schwartz JM, Baxter LR (1998): Neuroimaging and frontal-subcortical circuitry in obsessive-compulsive disorder. Brit J Psychiat 173:26-38

Schwartz JM, Stoessel PW, Baxter LR, Martin KM, Phelps ME (1996): Systematic changes in cerebral glucose metabolic rate after successful behavior modification treatment of obsessive-compulsive disorder. Arch Gen Psychiat 53:109-113

Shink E, Bevan MD, Bolam JP, Smith Y (1996): The subthalamic nucleus and the external pallidum two tightly interconnected structures that control the output of the basal ganglia in the monkey. Neuroscience 73:335-357

Small GW, Stern CE, Mandelkern MA, Fairbanks LA, Min CA, Guze BH (1992): Reliability of drawing regions of interest for positron emission tomography. Psych Res 45:177-185

Smith Y, Hazrati L-N, Parent A (1990): Efferent projections of the subthalamic nucleus in the squirrel monkey as studied by the PHA-L anterograde tracing method. J Comp Neurol 294:306-323

Spitzer RL, Endicott J (1978): Schedule for Affective Disorders and Schizophrenia. New York, New York State Psychiatric Institute

Swedo S, Schapiro MG, Grady CL, Cheslow DL, Leonard HL, Kumar A, Friedland R, Rapaport SL, Rappaport JL (1989): Cerebral glucose metabolism in childhood onset obsessive-compulsive disorder. Arch Gen Psychiatry 46:518-523

Swerdlow, NR (1995): Serotonin, obsessive-compulsive disorder, and the basal ganglia. Int Rev Psychiat 7:115-129

Wood A, Tollefson CD, Birkett M (1993): Phannacotherapy of obsessive-compulsive disorder-Experience with fluoxetine. Int Clin Psychopharmacol 8:301-306

Zald DH, Kim SW (1996a): Anatomy and function of the orbital frontal cortex, I: Anatomy, neurocircuitry, and obsessive-compulsive disorder. J Neuropsychiat Clin Neurosci 8:125-138

Zald DH, Kim SW (1996b): Anatomy and function of the orbital frontal cortex, II: Function and relevance to obsessive-compulsive disorder. J Neuropsychiat Clin Neurosci 8:249-261

Zohar J, Judge R (1996): Paroxetine versus clomipramine in the treatment of obsessive-compulsive disorder. OCD Paroxetine Study Investigators. Brit J Psychiat 169:468-74 